\title{
UNIQUENESS FOR AN INVERSE COEFFICIENT PROBLEM FOR A ONE-DIMENSIONAL TIME-FRACTIONAL DIFFUSION EQUATION WITH NON-ZERO BOUNDARY CONDITIONS
}

\author{
${ }^{1}$ WILLIAM RUNDELL ${ }^{2,3,4}$ MASAHIRO YAMAMOTO
}

\begin{abstract}
We consider initial boundary value problems for one-dimensional diffusion equation with time-fractional derivative of order $\alpha \in(0,1)$ which are subject to non-zero Neumann boundary conditions. We prove the uniqueness for an inverse coefficient problem of determining a spatially varying potential and the order of the time-fractional derivative by Dirichlet data at one end point of the spatial interval. The imposed Neumann conditions are required to be within the correct Sobolev space of order $\alpha$. Our proof is based on a representation formula of solution to an initial boundary value problem with non-zero boundary data. Moreover, we apply such a formula and prove the uniqueness in the determination of boundary value at another end point by Cauchy data at one end point.
\end{abstract}

Key words. inverse coefficient problem, fractional diffusion equation, uniqueness

AMS subject classifications. 35R30, 35R 11

\section{INTRODUCTION}

We consider the following initial boundary value problem for a one-dimensional timefractional diffusion equation:

$$
\left\{\begin{array}{l}
d_{t}^{\alpha} u(x, t)=\partial_{x}^{2} u(x, t)+p(x) u(x, t), \quad 0<x<1,0<t<T \\
\partial_{x} u(0, t)=0, \quad \partial_{x} u(1, t)=g(t), \quad 0<t<T \\
u(x, 0)=0, \quad 0<x<1 .
\end{array}\right.
$$

1 Department of Mathematics, Texas A\&M University, College Station, Texas 77843 USA e-mail: rundell@math. tamu.edu

${ }^{2}$ Graduate School of Mathematical Sciences, The University of Tokyo, Komaba, Meguro, Tokyo 153-8914, Japan

3 Honorary Member of Academy of Romanian Scientists, Splaiul Independentei Street, no 54, 050094 Bucharest Romania

${ }^{4}$ Peoples' Friendship University of Russia (RUDN University) 6 Miklukho-Maklaya St, Moscow, 117198, Russian Federation e-mail: myama@ms.u-tokyo.ac.jp . 
Here and henceforth let $\partial_{x}=\frac{\partial}{\partial x}, \partial_{x}^{2}=\frac{\partial^{2}}{\partial x^{2}}$, and we define for absolutely continuous $g$ on $[0, T]$

$$
d_{t}^{\alpha} g(t)=\frac{1}{\Gamma(1-\alpha)} \int_{0}^{t}(t-s)^{-\alpha} \frac{d g}{d s}(s) d s, \quad 0<t<T,
$$

that is, the fractional derivative of order $\alpha, 0<\alpha<1$, and of Caputo type (see, for example, Podlubny [24]). The first equation in (1.1) is a time-fractional diffusion equation of subdiffusion type modelling, for example, anomalous diffusion in heterogeneous media. For some applications see, for example, Metzler and Klafter [21].

In this article we are concerned with the question of uniqueness for the inverse problem: Let $g=g(t)$ be given for $0<t<T$. Given data $u(0, t)$ for $0<t<T$ or $u(1, t)$ for $0<t<T$, does this uniquely determine $\alpha \in(0,1)$ and $p(x), 0<x<1$ ?

In place of (1.1) we can also consider

$$
\left\{\begin{array}{l}
d_{t}^{\alpha} u(x, t)=\partial_{x}^{2} u(x, t)+p(x) u(x, t), \quad 0<x<1,0<t<T, \\
\partial_{x} u(0, t)=\partial_{x} u(1, t)=0, \quad 0<t<T \\
u(x, 0)=a(x), \quad 0<x<1 .
\end{array}\right.
$$

Uniqueness for this type of inverse problem for (1.2) with $\alpha=1$, that is, for the initial boundary value problem for the heat equation, was considered by, for example, Murayama [22, Suzuki and Murayama [27]. For the case with $0<\alpha<1$, we refer to Cheng, Nakagawa, Yamamoto and Yamazaki [3], Li, Zhang, Jia and Yamamoto [13]. Also see Jin and Rundell [7], Jing and Yamamoto 8, and survey chapters Li, Liu and Yamamoto [14], Li and Yamamoto [16], Liu, Li and Yamamoto [19]. Both for the cases of $\alpha=1$ and $0<\alpha<1$, the uniqueness for (1.2) requires a quite strong condition to be imposed for the initial value $a(x)$.

On the other hand, for the inverse problem for (1.1) with a zero initial value but $g \not \equiv 0$, we refer to Pierce [23] who proved the uniqueness for $\alpha=1$ with the quite mild assumption $g \not \equiv 0$.

For fixed $\alpha \in(1,2)$, Wei and Yan [29] established the uniqueness in determining $p(x)$ with $g \in C^{2}[0, T]$ imposing additional conditions.

For the inverse problem for (1.1) with $0<\alpha<1$, see Rundell and Yamamoto [25]. The purpose of this article is to complete [25] within a weaker class of solutions in suitable Sobolev 
space in time. For the case of $1<\alpha<2$, we can argue in a similar manner but we concentrate on the case $0<\alpha<1$.

For the mathematical formulations, we need to introduce function spaces and relevant operators; all functions considered are assumed to be real-valued. Let $L^{2}(0,1)$ be a usual Lebesgue space and let $\langle\cdot, \cdot\rangle$ and $\|\cdot\|$ denote the scalar product and the norm respectively in $L^{2}(0,1)$, and let $\langle\cdot, \cdot\rangle_{X}$ be the scalar product in other Hilbert spaces $X$ when we so specify.

We define the fractional Sobolev space $H^{\alpha}(0, T)$ on the interval $(0, T)$ (see e.g., [1], Chapter VII) with the norm in $H^{\alpha}(0, T)$ :

$$
\|u\|_{H^{\alpha}(0, T)}:=\left(\|u\|_{L^{2}(0, T)}^{2}+\int_{0}^{T} \int_{0}^{T} \frac{|u(t)-u(s)|^{2}}{|t-s|^{1+2 \alpha}} d t d s\right)^{\frac{1}{2}} .
$$

We further define the Banach spaces

$$
H_{\alpha}(0, T):=\left\{\begin{array}{l}
\left\{u \in H^{\alpha}(0, T) ; u(0)=0\right\}, \quad \frac{1}{2}<\alpha<1, \\
\left\{v \in H^{\frac{1}{2}}(0, T) ; \int_{0}^{T} \frac{|v(t)|^{2}}{t} d t<\infty\right\}, \quad \alpha=\frac{1}{2}, \\
H^{\alpha}(0, T), \quad 0<\alpha<\frac{1}{2}
\end{array}\right.
$$

with the following norm:

$$
\|v\|_{H_{\alpha}(0, T)}=\left\{\begin{aligned}
\|v\|_{H^{\alpha}(0, T)}, & 0<\alpha<1, \alpha \neq \frac{1}{2}, \\
\left(\|v\|_{H^{\frac{1}{2}(0, T)}}^{2}+\int_{0}^{T} \frac{|v(t)|^{2}}{t} d t\right)^{\frac{1}{2}}, & \alpha=\frac{1}{2} .
\end{aligned}\right.
$$

We define the Abel (Riemann-Liouville) fractional integral operator

$$
J^{\alpha} g(t)=\frac{1}{\Gamma(\alpha)} \int_{0}^{t}(t-s)^{\alpha-1} g(s) d s, \quad 0<t<T, \quad 0<\alpha<1 .
$$

Henceforth by $x \sim y$, we mean that there exists a constant $C>0$ such that $C^{-1} y \leq x \leq C y$ for all quantities $x, y$ under consideration.

In Gorenflo, Luchko and Yamamoto [6], Kubica, Ryszewska and Yamamoto [10] (Theorem $2.1)$, it is proved that $J^{\alpha}$ is an isomorphism between $L^{2}(0, T)$ and $H_{\alpha}(0, T)$. We define

$$
\partial_{t}^{\alpha} g=\left(J^{\alpha}\right)^{-1} g \quad \text { for } g \in H_{\alpha}(0, T)=J^{\alpha} L^{2}(0, T)
$$

Then also by Theorem 2.5 in [10], we see

$$
\left\{\begin{array}{l}
\left\|\partial_{t}^{\alpha} g\right\|_{L^{2}(0, T)} \sim\|g\|_{H_{\alpha}(0, T)}, \quad g \in H_{\alpha}(0, T) \\
\partial_{t}^{\alpha} g=d_{t}^{\alpha} g \quad \text { if } g \in W^{1,1}(0, T) \text { satisfies } g(0)=0 \text { and } t^{\alpha-1} \frac{d g}{d t} \in L^{\infty}(0, T)
\end{array}\right.
$$


In other words, $\partial_{t}^{\alpha}$ is an extension of the Caputo derivative $d_{t}^{\alpha}$ to $H_{\alpha}(0, T)$.

Thus throughout this article, in place of (1.1) we consider

$$
\left\{\begin{array}{l}
\partial_{t}^{\alpha} u(x, t)=\partial_{x}^{2} u(x, t)+p(x) u(x, t), \quad 0<x<1,0<t<T, \\
\partial_{x} u(0, t)=0, \quad \partial_{x} u(1, t)=g(t), \quad 0<t<T \\
u \in H_{\alpha}\left(0, T ; L^{2}(0,1)\right) .
\end{array}\right.
$$

We assume

$$
p, q \leq 0 \quad \text { on }[0,1], \quad p, q \in C[0,1]
$$

Then we can prove

\section{Proposition 1.}

Let $g \in H_{\alpha}(0, T)$ and let $0<\alpha<1$. Then there exists a unique solution $u_{p, \alpha}=u_{p, \alpha}(x, t) \in$ $H_{\alpha}\left(0, T ; L^{2}(0,1)\right) \cap L^{2}\left(0, T ; H^{2}(0,1)\right)$ solving (1.4) .

In (1.4), we interpret $u(x, \cdot) \in H_{\alpha}(0, T)$ as an initial condition: if $\alpha>\frac{1}{2}$, then the Sobolev embedding yields $H_{\alpha}\left(0, T ; L^{2}(0,1)\right) \subset H^{\alpha}\left(0, T ; L^{2}(0,1)\right) \subset C\left([0, T] ; L^{2}(0,1)\right)$ and so this

means that $u$ satisfies the initial condition in a usual sense. However for $\alpha<\frac{1}{2}$, the time regularity does not admit such a usual initial condition and alternatively the third equation in (1.4) is required. For the class of solutions with the $H_{\alpha}$-regularity in $t$, it is sufficient to assume the same regularity in $t$ for boundary data $g(t)$, that is, $g \in H_{\alpha}(0, T)$. Moreover for $\alpha>\frac{1}{2}$, the condition means that $g(0)=0$, which is a natural compatibility condition at $x=0$ and $t=0$. We emphasize that since the order of time derivative appearing in the equation is up to $\alpha<1$, it is natural to work within " $\alpha$-time differentiability", and not in the $C^{1}$ nor $H^{1}$-class.

For the initial boundary value problems with the zero boundary values, we refer to Gorenflo, Luchko and Yamamoto [6], Kian and Yamamoto [9], Kubica, Ryszewska and Yamamoto [10], Kubica and Yamamoto [11], Luchko [20], Sakamoto and Yamamoto [26]. On the other hand, for initial boundary value problems with non-zero boundary data, there are not many works and we refer only to Yamamoto [30] in the case of less regular boundary data, and one can consult the references therein. On the other hand, the proof of Proposition 1 can be done directly, thanks to the one-dimensionality, and see Section 2. 
Now we are ready to state the main result of this article.

Theorem 2. We assume (1.5) and $0<\alpha, \beta<1$,

$$
g \in H_{\max \{\alpha, \beta\}}(0, T), \quad g \not \equiv 0 \quad \text { in }(0, T) .
$$

Then either $u_{p, \alpha}(0, t)=u_{q, \beta}(0, t)$ for $0<t<T$ or $u_{p, \alpha}(1, t)=u_{q, \beta}(1, t)$ for $0<t<T$, yields

$$
\alpha=\beta, \quad p(x)=q(x), \quad 0<x<1 .
$$

By the regularity shown in Proposition 1 and the trace theorem, we notice that the data $u_{p, \alpha}(0, t)$, etc. can make sense in $L^{2}(0, T)$. We stress that the condition $g \not \equiv 0$ in (1.6) for the boundary input is quite generous.

The article is composed of four sections. In Section 2, we prove Proposition 1 and a key representation formula of the solution $u_{p, \alpha}$ to (1.4). Section 3 is devoted to the proof of Theorem 2 on the basis of the representation formula in Section 2. In Section 4, we provide one application of the representation formula to prove the uniqueness in determining a boundary value at $x=1$ by by Cauchy data at $x=0$.

\section{Proof of Proposition 1 And a Representation Formula}

\subsection{Proof of Proposition 1.}

Recalling (1.5) we define an operator $A_{p}$ in $L^{2}(0,1)$ by

$$
\left\{\begin{array}{l}
A_{p} w(x)=-\frac{d^{2} w}{d x^{2}}(x)-p(x) w(x), \quad 0<x<1 \\
\mathcal{D}\left(A_{p}\right)=\left\{w \in H^{2}(0,1) ; \frac{d w}{d x}(0)=\frac{d w}{d x}(1)=0\right\}
\end{array}\right.
$$

Then $A_{p}$ possesses eigenvalues $0<\lambda_{1}<\lambda_{2}<\cdots$. Let $\varphi_{n}, n \in \mathbb{N}$ be the associated unique eigenfunction for $\lambda_{n}: \varphi_{n} \in \mathcal{D}\left(A_{p}\right)$ satisfies $A_{p} \varphi_{n}=\lambda_{n} \varphi_{n}$ in $(0,1)$ and we make the normalisation $\varphi_{n}(1)=1$. Moreover, it is known that $\left\langle\varphi_{n}, \varphi_{m}\right\rangle:=\int_{\Omega} \varphi_{n}(x) \varphi_{m}(x) d x=0$ for $n \neq m$ and we set the associated norming constants as

$$
\rho_{n}:=\left\|\varphi_{n}\right\|^{2}, \quad n \in \mathbb{N}
$$


We define

$$
\left\{\begin{array}{l}
v(x, t)=u_{p, \alpha}(x, t)-\frac{x^{2}}{2} g(t), \\
f(x, t)=-\frac{x^{2}}{2} \partial_{t}^{\alpha} g(t)+g(t)+\frac{x^{2}}{2} p(x) g(t), \quad 0<x<1,0<t<t .
\end{array}\right.
$$

Then (1.4) is equivalent to

$$
\left\{\begin{array}{l}
\partial_{t}^{\alpha} v(x, t)=\partial_{x}^{2} v(x, t)+p(x) v(x, t)+f(x, t), \quad 0<x<1,0<t<T, \\
\partial_{x} v(0, t)=\partial_{x} v(1, t)=0, \quad 0<t<T, \\
v \in H_{\alpha}\left(0, T ; L^{2}(0,1)\right) .
\end{array}\right.
$$

Since $g \in H_{\alpha}(0, T)$, we see that $v \in H_{\alpha}\left(0, T ; L^{2}(0,1)\right)$ if and only if $u \in H_{\alpha}\left(0, T ; L^{2}(0,1)\right)$.

From $g \in H_{\alpha}(0, T)$ and $p \in C[0,1]$, it follows that $f \in L^{2}\left(0, T ; L^{2}(0,1)\right)$. Thus it is sufficient to prove the unique existence of solution $v \in H_{\alpha}\left(0, T ; L^{2}(0,1)\right) \cap L^{2}\left(0, T ; H^{2}(0,1)\right)$ to (2.2). This follows from [10], 26] for example. We note that in [10], [26], the zero Dirichlet boundary condition is considered and the case of he zero Neumann boundary condition can be treated in the same way. Thus the proof of Proposition 1 is complete.

\subsection{The representation formula.}

For $\gamma_{1}, \gamma_{2}>0$, we define the two parameter Mittag-Leffler function:

$$
E_{\gamma_{1}, \gamma_{2}}(z)=\sum_{k=0}^{\infty} \frac{z^{k}}{\Gamma\left(\gamma_{1} k+\gamma_{2}\right)}, \quad z \in \mathbb{C} .
$$

This is an entire function of order 1 in $z \in \mathbb{C}$ (e.g., Gorenflo, Kilbas, Mainardi and Rogosin [5], Podlubny [24]). Then

\section{Proposition 3 (representation formula).}

Let $0<\alpha<1, p$ satisfy (1.5) and $g \in H_{\alpha}(0, T)$. Then

$$
u_{p, \alpha}(x, t)=\sum_{n=1}^{\infty} \frac{1}{\rho_{n}}\left(\int_{0}^{t}(t-s)^{\alpha-1} E_{\alpha, \alpha}\left(-\lambda_{n}(t-s)^{\alpha}\right) g(s) d s\right) \varphi_{n}(x)
$$

in $H_{\alpha}\left(0, T ; L^{2}(0,1)\right) \cap L^{2}\left(0, T ; H^{2}(0,1)\right)$.

\section{Proof of Proposition 3.}

By [26] for example, we have the representation

$$
v(x, t)=\sum_{n=1}^{\infty} \frac{1}{\rho_{n}}\left(\int_{0}^{t}(t-s)^{\alpha-1} E_{\alpha, \alpha}\left(-\lambda_{n}(t-s)^{\alpha}\right)\left\langle f(\cdot, s), \varphi_{n}\right\rangle d s\right) \varphi_{n}(x)
$$


in $H_{\alpha}\left(0, T ; L^{2}(0,1)\right) \cap L^{2}\left(0, T ; H^{2}(0,1)\right)$. Here we note equations (2.1) and (2.2).

We set ${ }_{0} C^{1}[0, T]:=\left\{h \in C^{1}[0, T] ; h(0)=0\right\}$.

First we prove (2.3) for $g \in{ }_{0} C^{1}[0, T]$. We have to calculate the right-hand side of (2.4).

$$
\begin{aligned}
\int_{0}^{t}(t & -s)^{\alpha-1} E_{\alpha, \alpha}\left(-\lambda_{n}(t-s)^{\alpha}\right)\left\langle f(\cdot, s), \varphi_{n}\right\rangle d s \\
= & -\int_{0}^{t}(t-s)^{\alpha-1} E_{\alpha, \alpha}\left(-\lambda_{n}(t-s)^{\alpha}\right) \partial_{s}^{\alpha} g(s) d s\left\langle\frac{x^{2}}{2}, \varphi_{n}\right\rangle \\
& +\int_{0}^{t}(t-s)^{\alpha-1} E_{\alpha, \alpha}\left(-\lambda_{n}(t-s)^{\alpha}\right) g(s) d s\left\langle 1+\frac{x^{2}}{2} p, \varphi_{n}\right\rangle .
\end{aligned}
$$

We set

$$
S:=\int_{0}^{t}(t-s)^{\alpha-1} E_{\alpha, \alpha}\left(-\lambda_{n}(t-s)^{\alpha}\right) \partial_{s}^{\alpha} g(s) d s .
$$

For $g \in{ }_{0} C^{1}[0, T]$, by (1.3) we see that $\partial_{s}^{\alpha} g$ coincides with $d_{s}^{\alpha} g$ :

$$
\partial_{s}^{\alpha} g(s)=d_{s}^{\alpha}(s)=\frac{1}{\Gamma(1-\alpha)} \int_{0}^{s}(s-\xi)^{-\alpha} \frac{d g}{d \xi}(\xi) d \xi .
$$

Therefore, change of the order of integration yields

$$
\begin{aligned}
& S=\frac{1}{\Gamma(1-\alpha)} \int_{0}^{t}(t-s)^{\alpha-1} E_{\alpha, \alpha}\left(-\lambda_{n}(t-s)^{\alpha}\right)\left(\int_{0}^{s}(s-\xi)^{-\alpha} \frac{d g}{d \xi}(\xi) d \xi\right) d s \\
& =\frac{1}{\Gamma(1-\alpha)} \int_{0}^{t} \frac{d g}{d \xi}(\xi)\left(\int_{\xi}^{t}(t-s)^{\alpha-1} E_{\alpha, \alpha}\left(-\lambda_{n}(t-s)^{\alpha}\right)(s-\xi)^{-\alpha} d s\right) d \xi \\
& =\int_{0}^{t} \frac{d g}{d \xi}(\xi) \frac{1}{\Gamma(1-\alpha)}\left(\int_{0}^{t-\xi} \eta^{\alpha-1} E_{\alpha, \alpha}\left(-\lambda_{n} \eta^{\alpha}\right)(t-\xi-\eta)^{-\alpha} d \eta\right) d \xi .
\end{aligned}
$$

For the last equality we used the change of variables $s \rightarrow \eta$ by $\eta=t-s$. Moreover,

$$
\frac{1}{\Gamma(1-\alpha)} \int_{0}^{t-\xi} \eta^{\alpha-1} E_{\alpha, \alpha}\left(-\lambda_{n} \eta^{\alpha}\right)(t-\xi-\eta)^{-\alpha} d \eta=E_{\alpha, 1}\left(-\lambda_{n}(t-\xi)^{\alpha}\right)
$$

(e.g., formula (1.100) (p.25) in [24]). Hence, again applying integration by parts, we obtain

$$
\begin{aligned}
S & =\int_{0}^{t} \frac{d g}{d \xi}(\xi) E_{\alpha, 1}\left(-\lambda_{n}(t-\xi)^{\alpha}\right) d \xi \\
& =\left[g(\xi) E_{\alpha, 1}\left(-\lambda_{n}(t-\xi)^{\alpha}\right)\right]_{\xi=0}^{\xi=t}-\int_{0}^{t} g(\xi) \frac{d}{d \xi} E_{\alpha, 1}\left(-\lambda_{n}(t-\xi)^{\alpha}\right) d \xi
\end{aligned}
$$

Now, by the definition of the Mittag-Lefler function in view of the power series, the termwise differentiation yields

$$
\frac{d}{d \xi} E_{\alpha, 1}\left(-\lambda_{n}(t-\xi)^{\alpha}\right)=\lambda_{n}(t-\xi)^{\alpha-1} E_{\alpha, \alpha}\left(-\lambda_{n}(t-\xi)^{\alpha}\right), \quad 0<\xi<t<T .
$$


Therefore, using $g(0)=0$ by $g \in{ }_{0} C^{1}[0, T]$, we have

$$
S=g(t)-\int_{0}^{t} \lambda_{n}(t-s)^{\alpha-1} E_{\alpha, \alpha}\left(-\lambda_{n}(t-s)^{\alpha}\right) g(s) d s .
$$

Substituting this into the above we obtain

$$
\begin{aligned}
& \int_{0}^{t}(t-s)^{\alpha-1} E_{\alpha, \alpha}\left(-\lambda_{n}(t-s)^{\alpha}\right)\left\langle f(\cdot, s), \varphi_{n}\right\rangle s d s=-g(t)\left\langle\frac{x^{2}}{2}, \varphi_{n}\right\rangle \\
+ & \int_{0}^{t}(t-s)^{\alpha-1} E_{\alpha, \alpha}\left(-\lambda_{n}(t-s)^{\alpha}\right) g(s) d s\left(\left\langle\lambda_{n} \frac{x^{2}}{2}, \varphi_{n}\right\rangle+\left\langle 1+\frac{x^{2}}{2} p, \varphi_{n}\right\rangle\right) .
\end{aligned}
$$

Here by integration by parts, we calculate

$$
\begin{aligned}
& \left\langle\lambda_{n} \frac{x^{2}}{2}, \varphi_{n}\right\rangle+\left\langle\frac{x^{2}}{2} p, \varphi_{n}\right\rangle=\left\langle\lambda_{n} \varphi_{n}+p \varphi_{n}, \frac{x^{2}}{2}\right\rangle \\
& =\left\langle-\frac{d^{2} \varphi_{n}}{d x^{2}}, \frac{x^{2}}{2}\right\rangle=\left[-\frac{d \varphi_{n}}{d x}(x) \frac{x^{2}}{2}\right]_{x=0}^{x=1}+\int_{0}^{1} x \frac{d \varphi_{n}}{d x}(x) d x \\
& =\left[x \varphi_{n}(x)\right]_{x=0}^{x=1}-\int_{0}^{1} \varphi_{n}(x) d x=1-\left\langle\varphi_{n}, 1\right\rangle .
\end{aligned}
$$

Hence

$$
\left\langle\lambda_{n} \frac{x^{2}}{2}, \varphi_{n}\right\rangle+\left\langle 1+\frac{x^{2}}{2} p, \varphi_{n}\right\rangle=1-\left\langle\varphi_{n}, 1\right\rangle+\left\langle 1, \varphi_{n}\right\rangle=1
$$

so that

$$
\begin{aligned}
\int_{0}^{t}(t & -s)^{\alpha-1} E_{\alpha, \alpha}\left(-\lambda_{n}(t-s)^{\alpha}\right)\left\langle f(\cdot, s), \varphi_{n}\right\rangle d s \\
& =-\left\langle\frac{x^{2}}{2}, \varphi_{n}\right\rangle g(t)+\int_{0}^{t}(t-s)^{\alpha-1} E_{\alpha, \alpha}\left(-\lambda_{n}(t-s)^{\alpha}\right) g(s) d s .
\end{aligned}
$$

Substituting this into (2.4), since $\left\{\frac{1}{\sqrt{\rho_{n}}} \varphi_{n}\right\}_{n \in \mathbb{N}}$ is an orthonormal basis in $L^{2}(0,1)$, we see

$$
\begin{aligned}
& v(x, t)=-\sum_{n=1}^{\infty} \frac{1}{\rho_{n}}\left\langle\frac{x^{2}}{2}, \varphi_{n}\right\rangle g(t) \varphi_{n}(x)+\sum_{n=1}^{\infty} \frac{1}{\rho_{n}} \int_{0}^{t}(t-s)^{\alpha-1} E_{\alpha, \alpha}\left(-\lambda_{n}(t-s)^{\alpha}\right) g(s) d s \varphi_{n}(x) \\
& =-\frac{x^{2}}{2} g(t)+\sum_{n=1}^{\infty} \frac{1}{\rho_{n}}\left(\int_{0}^{t}(t-s)^{\alpha-1} E_{\alpha, \alpha}\left(-\lambda_{n}(t-s)^{\alpha}\right) g(s) d s\right) \varphi_{n}(x) .
\end{aligned}
$$

Since $u=v+\frac{x^{2}}{2} g(t)$, we have proved (2.3) for $g \in{ }_{0} C^{1}[0, T]$.

Next we have to prove (2.3) for $g \in H_{\alpha}(0, T)$. In equations (2.1) and (2.4), we write $f:=f_{g}$ and $v:=v_{g}$ respectively in order to specify the dependence on $g$. Since ${ }_{0} C^{1}[0, T]:=\{h \in$ $\left.C^{1}[0, T] ; h(0)=0\right\}$ is dense in $H_{\alpha}(0, T)$ (e.g., Lemma 2.2 in [10]), for each $g \in H_{\alpha}(0, T)$, we can find a sequence $g_{\ell} \in{ }_{0} C^{1}[0, T], \ell \in \mathbb{N}$ such that $g_{\ell} \longrightarrow g$ in $H_{\alpha}(0, T)$. Then, since $\partial_{t}^{\alpha} g_{\ell} \longrightarrow \partial_{t}^{\alpha} g$ in $L^{2}(0, T)$ (e.g., Theorem 2.4 in [10]), it follows that $f_{g_{\ell}} \longrightarrow f_{g}$ in $L^{2}(0, T)$. 
Therefore, applying the well-posedness for the initial boundary value problem (e.g., 6], Theorem 4.1 in [10], [26]), we see that $v_{g_{\ell}} \longrightarrow v_{g}$ in $H_{\alpha}\left(0, T ; L^{2}(0,1)\right) \cap L^{2}\left(0, T ; H^{2}(0,1)\right)$.

As we already proved for $g_{\ell} \in{ }_{0} C^{1}[0, T]$, we have

$$
\sum_{n=1}^{\infty} \frac{1}{\rho_{n}} \int_{0}^{t}(t-s)^{\alpha-1} E_{\alpha, \alpha}\left(-\lambda_{n}(t-s)^{\alpha}\right) g_{\ell}(s) d s \varphi_{n}-\frac{x^{2}}{2} g_{\ell}(t) \longrightarrow v_{g}
$$

in the space $H_{\alpha}\left(0, T ; L^{2}(0,1)\right) \cap L^{2}\left(0, T ; H^{2}(0,1)\right)$.

On the other hand, let $h \in L^{2}(0, T)$. Then one can prove by the asymptotic behavior of $\varphi_{n}$ for large $n \in \mathbb{N}$ (e.g., Section 2 of Chapter 1 of Levitan and Sargsjan [12]), that there exists a constant $\rho_{0}>0$ such that

$$
\rho_{n} \geq \rho_{0} \quad \text { for all } n \in \mathbb{N} \text {. }
$$

Henceforth $C>0$ denotes generic constants which are independent of $n$ and choices of $h, g, t \in(0, T)$. Let $\psi \in C_{0}^{\infty}((0,1) \times(0, T))$. Then by integration by parts

$$
\left\langle\varphi_{n}, \psi(\cdot, s)\right\rangle=\frac{1}{\lambda_{n}}\left\langle\lambda_{n} \varphi_{n}, \psi(\cdot, s)\right\rangle=\frac{1}{\lambda_{n}}\left\langle A_{p} \varphi_{n}, \psi(\cdot, s)\right\rangle=\frac{1}{\lambda_{n}}\left\langle\varphi_{n}, A_{p} \psi(\cdot, s)\right\rangle .
$$

Therefore,

$$
\begin{aligned}
& \left\langle\sum_{n=1}^{\infty} \frac{1}{\rho_{n}}\left(\int_{0}^{t}(t-s)^{\alpha-1} E_{\alpha, \alpha}\left(-\lambda_{n}(t-s)^{\alpha}\right) h(s) d s\right) \varphi_{n}, \psi\right\rangle_{L^{2}((0,1) \times(0, T))} \\
& \quad=\sum_{n=1}^{\infty} \frac{1}{\rho_{n}}\left\langle\int_{0}^{t}(t-s)^{\alpha-1} E_{\alpha, \alpha}\left(-\lambda_{n}(t-s)^{\alpha}\right) h(s) d s, \frac{1}{\lambda_{n}}\left\langle\varphi_{n}, A_{p} \psi(\cdot, t)\right\rangle_{L^{2}(0,1)}\right\rangle_{L^{2}(0, T)} .
\end{aligned}
$$

Hence, also by (2.8) and the Cauchy-Schwarz inequality, we have

$$
\begin{aligned}
& \left|\left\langle\sum_{n=1}^{\infty} \frac{1}{\rho_{n}}\left(\int_{0}^{t}(t-s)^{\alpha-1} E_{\alpha, \alpha}\left(-\lambda_{n}(t-s)^{\alpha}\right) h(s) d s\right) \varphi_{n}, \psi\right\rangle_{L^{2}((0,1) \times(0, T))}\right| \\
& \quad \leq C \sum_{n=1}^{\infty} \frac{1}{\lambda_{n}}\left\|\int_{0}^{t}(t-s)^{\alpha-1} E_{\alpha, \alpha}\left(-\lambda_{n}(t-s)^{\alpha}\right) h(s) d s\right\|_{L^{2}(0, T)}\left\|A_{p} \psi\right\|_{L^{2}\left(0, T ; L^{2}(0,1)\right)} \frac{\left\|\varphi_{n}\right\|}{\rho_{n}} \\
& \leq C \sum_{n=1}^{\infty} \frac{1}{\lambda_{n}}\left\|s^{\alpha-1} E_{\alpha, \alpha}\left(-\lambda_{n} s^{\alpha}\right) * h\right\|_{L^{2}(0, T)} .
\end{aligned}
$$

Here and henceforth we set $\left(g_{1} * g_{2}\right)(t):=\int_{0}^{t} g_{1}(t-s) g_{2}(s) d s$. By a bound of $E_{\alpha, \alpha}\left(-\lambda_{n} s^{\alpha}\right)$ (e.g., Theorem 1.6 (p.35) in [24]), we have $\left|E_{\alpha, \alpha}\left(-\lambda_{n} s^{\alpha}\right)\right| \leq C$ for all $n \in \mathbb{N}$ and $s>0$. Hence, Young's inequality yields

$$
\left\|s^{\alpha-1} E_{\alpha, \alpha}\left(-\lambda_{n} s^{\alpha}\right) * h\right\|_{L^{2}(0, T)} \leq\left\|s^{\alpha-1} E_{\alpha, \alpha}\left(-\lambda_{n} s^{\alpha}\right)\right\|_{L^{1}(0, T)}\|h\|_{L^{2}(0, T)} \leq C\|h\|_{L^{2}(0, T)} .
$$


Since $C^{-1} n^{2} \leq \lambda_{n} \leq C n^{2}$ for all $n \in \mathbb{N}$ (e.g., [12]), we can obtain

$$
\begin{aligned}
& \left|\left\langle\sum_{n=1}^{\infty} \frac{1}{\rho_{n}}\left(\int_{0}^{t}(t-s)^{\alpha-1} E_{\alpha, \alpha}\left(-\lambda_{n}(t-s)^{\alpha}\right) h(s) d s\right) \varphi_{n}, \psi\right\rangle_{L^{2}((0,1) \times(0, T))}\right| \\
& \quad \leq C \sum_{n=1}^{\infty} \frac{1}{n^{2}}\|h\|_{L^{2}(0, T)} \leq C\|h\|_{L^{2}(0, T)}
\end{aligned}
$$

for all $\psi \in C_{0}^{\infty}((0,1) \times(0, T))$.

Therefore, setting $h:=g-g_{\ell}$, we see that

$$
\begin{aligned}
& \sum_{n=1}^{\infty} \frac{1}{\rho_{n}} \int_{0}^{t}(t-s)^{\alpha-1} E_{\alpha, \alpha}\left(-\lambda_{n}(t-s)^{\alpha}\right) g_{\ell}(s) d s \varphi_{n}-\frac{x^{2}}{2} g_{\ell}(t) \\
& \quad \longrightarrow \sum_{n=1}^{\infty} \frac{1}{\rho_{n}} \int_{0}^{t}(t-s)^{\alpha-1} E_{\alpha, \alpha}\left(-\lambda_{n}(t-s)^{\alpha}\right) g(s) d s \varphi_{n}-\frac{x^{2}}{2} g(t) \quad \text { in }\left(C_{0}^{\infty}((0,1) \times(0, T))\right)^{\prime} .
\end{aligned}
$$

In view of (2.7), the convergence is in $H_{\alpha}\left(0, T ; L^{2}(0,1)\right) \cap L^{2}\left(0, T ; H^{2}(0,1)\right)$, and both limits in (2.7) and the above must coincide. Hence,

$$
v_{g}(x, t)=\sum_{n=1}^{\infty} \frac{1}{\rho_{n}} \int_{0}^{t}(t-s)^{\alpha-1} E_{\alpha, \alpha}\left(-\lambda_{n}(t-s)^{\alpha}\right) g(s) d s \varphi_{n}-\frac{x^{2}}{2} g(t)
$$

in $H_{\alpha}\left(0, T ; L^{2}(0,1)\right) \cap L^{2}\left(0, T ; H^{2}(0,1)\right)$. Since $u_{p, \alpha}(x, t)=v_{g}(x, t)+\frac{x^{2}}{2} g(t)$ by (2.1), the proof of Proposition 3 is complete.

We conclude this section with the following lemma.

\section{Lemma 4.}

Let $K_{p, \alpha}(x, t)$ be defined by

$$
\begin{gathered}
K_{p, \alpha}(x, t):=\sum_{n=1}^{\infty} \frac{\varphi_{n}(x)}{\rho_{n}} \int_{0}^{t} s^{\alpha-1} E_{\alpha, \alpha}\left(-\lambda_{n} s^{\alpha}\right) d s=\sum_{n=1}^{\infty} \frac{\varphi_{n}(x)}{\lambda_{n} \rho_{n}}\left(1-E_{\alpha, 1}\left(-\lambda_{n} t^{\alpha}\right)\right) \\
\text { for all } x \in[0,1] \text { and } t \in[0, T] .
\end{gathered}
$$

Then,

(i) The series is uniform convergent in $x \in[0,1]$ and $t \in[0, T]$, and $K_{p, \alpha}(x, \cdot) \in L^{\infty}(0, \infty)$ and is analytic in $t>0$ for all $x \in[0,1]$.

(ii)

$$
\int_{0}^{\xi} u_{p, \alpha}(x, t) d t=\left(K_{p, \alpha}(x, \cdot) * g\right)(\xi) \quad \text { for all } x \in[0,1] \text { and } \xi \in[0, T] \text {. }
$$




\section{Proof of (i).}

In view of (2.6), we have

$$
\int_{0}^{t} s^{\alpha-1} E_{\alpha, \alpha}\left(-\lambda_{n} s^{\alpha}\right) d s=\frac{1}{\lambda_{n}} \int_{t}^{0} \frac{d}{d s}\left(E_{\alpha, 1}\left(-\lambda_{n} s^{\alpha}\right)\right) d s=\frac{1}{\lambda_{n}}\left(1-E_{\alpha, 1}\left(-\lambda_{n} t^{\alpha}\right)\right) .
$$

Hence,

$$
K_{p, \alpha}(x, t)=\sum_{n=1}^{\infty} \frac{1}{\lambda_{n} \rho_{n}}\left(1-E_{\alpha, 1}\left(-\lambda_{n} t^{\alpha}\right)\right) \varphi_{n}(x), \quad 0 \leq x \leq 1, t>0 .
$$

From Theorem 1.6 (p.35) in [24], we know that there exist constants $C>0$ and $\theta_{0}>0$ such that

$$
\left|E_{\alpha, 1}\left(-\lambda_{n} z^{\alpha}\right)\right| \leq C \quad \text { for all } n \in \mathbb{N} \text { and } z \in \Sigma:=\left\{z \in \mathbb{C} ;|\operatorname{Arg} z|<\theta_{0}\right\}
$$

We fix small $\delta>0$ arbitrarily. Since $\left\|\varphi_{n}\right\|_{H^{\theta}(0,1)} \leq C\left\|A_{p}^{\frac{\theta}{2}} \varphi_{n}\right\|_{L^{2}(0,1)}$ with $0<\theta<2$, applying the Sobolev embedding and recalling $\rho_{n}=\left\|\varphi_{n}\right\|_{L^{2}(0,1)}^{2}$, we have

$$
\left\|\varphi_{n}\right\|_{C[0,1]} \leq C\left\|\varphi_{n}\right\|_{H^{\frac{1}{2}+\delta}(0,1)} \leq C\left\|A_{p}^{\frac{1}{4}+\frac{\delta}{2}} \varphi_{n}\right\|_{L^{2}(0,1)}=C \lambda_{n}^{\frac{1}{4}+\frac{\delta}{2}} \sqrt{\rho_{n}} .
$$

Hence, by (2.8), we obtain

$$
\left|\frac{1}{\rho_{n} \lambda_{n}}\left(1-E_{\alpha, 1}\left(-\lambda_{n} z^{\alpha}\right)\right) \varphi_{n}(x)\right| \leq \frac{C}{\lambda_{n} \sqrt{\rho_{n}}} \lambda_{n}^{\frac{1}{4}+\frac{\delta}{2}}, \quad 0 \leq x \leq 1, z \in \Sigma,
$$

and so

$$
\begin{gathered}
\sum_{n=1}^{\infty} \frac{1}{\lambda_{n} \rho_{n}}\left|\left(1-E_{\alpha, 1}\left(-\lambda_{n} z^{\alpha}\right)\right) \varphi_{n}(x)\right| \\
\leq C \sum_{n=1}^{\infty} \frac{1}{\lambda_{n}^{\frac{3}{4}-\frac{\delta}{2}}} \leq C \sum_{n=1}^{\infty} \frac{1}{n^{\frac{3}{2}-\delta}}<\infty, \quad 0 \leq x \leq 1, z \in \Sigma .
\end{gathered}
$$

Here we used $\lambda_{n} \sim n^{2}$ (e.g., [12]). Since $E_{\alpha, 1}\left(-\lambda_{n} z^{\alpha}\right)$ is analytic in $z \in \Sigma$, we can complete the proof of $(\mathrm{i})$.

\section{Proof of (ii).}

Since the series in $(2.3)$ is convergent in $L^{2}\left(0, T ; H^{2}(0,1)\right)$, by $H^{2}(0,1) \subset C[0,1]$, we see that

$$
u_{p, \alpha}(x, t)=\int_{0}^{t}\left(\sum_{n=1}^{\infty} \frac{1}{\rho_{n}}(t-s)^{\alpha-1} E_{\alpha, \alpha}\left(-\lambda_{n}(t-s)^{\alpha}\right) \varphi_{n}(x)\right) g(s) d s
$$

is convergent in $L^{2}(0, T ; C[0, T])$. Therefore,

$$
\int_{0}^{\xi} u_{p, \alpha}(x, t) d t=\int_{0}^{\xi}\left\{\int_{0}^{t}\left(\sum_{n=1}^{\infty} \frac{1}{\rho_{n}}(t-s)^{\alpha-1} E_{\alpha, \alpha}\left(-\lambda_{n}(t-s)^{\alpha}\right) \varphi_{n}(x)\right) g(s) d s\right\} d t
$$


for all fixed $x \in[0,1]$. Exchanging the orders of the integrals and changing the variables $t \rightarrow \eta: \eta=t-s$, we obtain

$$
\begin{aligned}
& \int_{0}^{\xi}\left(\int_{0}^{t}(t-s)^{\alpha-1} E_{\alpha, \alpha}\left(-\lambda_{n}(t-s)^{\alpha}\right) g(s) d s\right) d t \\
= & \int_{0}^{\xi}\left(\int_{s}^{\xi}(t-s)^{\alpha-1} E_{\alpha, \alpha}\left(-\lambda_{n}(t-s)^{\alpha}\right) d t\right) g(s) d s=\int_{0}^{\xi}\left(\int_{0}^{\xi-s} \eta^{\alpha-1} E_{\alpha, \alpha}\left(-\lambda_{n} \eta^{\alpha}\right) d \eta\right) g(s) d s .
\end{aligned}
$$

Hence by (2.9), we have verified (ii) and the proof of Lemma 4 is complete.

\section{Proof of Theorem 2}

Let

$$
\left\{\begin{array}{l}
A_{q} w(x)=-\frac{d^{2} w}{d x^{2}}(x)-q(x) w(x), \quad 0<x<1, \\
\mathcal{D}\left(A_{q}\right)=\left\{w \in H^{2}(0,1) ; \frac{d w}{d x}(0)=\frac{d w}{d x}(1)=0\right\} .
\end{array}\right.
$$

We let $0<\mu_{1}<\mu_{2}<\cdots$, denote all the eigenvalues of the operator $A_{q}$ and let $\psi_{n}, n \in \mathbb{N}$ be the corresponding eigenfunction for $\mu_{n}$, that is $\psi_{n} \in \mathcal{D}\left(A_{q}\right)$ satisfies $A_{q} \psi_{n}=\mu_{n} \psi_{n}$ in $(0,1)$ and we take the normalization of the eigenfunctions to be $\psi_{n}(1)=1$. Given this, we set $\sigma_{n}:=\left\|\psi_{n}\right\|^{2}$, for $n \in \mathbb{N}$. Similarly to Lemma 4 , we define

$$
K_{q, \beta}(1, t)=\sum_{n=1}^{\infty} \frac{1}{\sigma_{n}} \int_{0}^{t} s^{\beta-1} E_{\beta, \beta}\left(-\mu_{n} s^{\beta}\right) d s, \quad t>0 .
$$

It is sufficient to prove the theorem with data $u_{p, \alpha}(1, t)=u_{q, \beta}(1, t), 0<t<T$. For the other case at $x=0$, replacing the conditions $\varphi_{n}(1)=\psi_{n}(1)=1$ by $\varphi_{n}(0)=\psi_{n}(0)=1$, we can repeat the whole argument and thus omit the details for this case.

Since $\int_{0}^{\xi} u_{p, \alpha}(1, t) d t=\int_{0}^{\xi} u_{q, \beta}(1, t) d t$ by $u_{p, \alpha}(1, t)=u_{q, \beta}(1, t)$ for $0<t<T$, in view of Lemma 4, we see

$$
\left(K_{p, \alpha}(1, \cdot) * g\right)(\xi)=\left(K_{q, \beta}(1, \cdot) * g\right)(\xi), \quad 0<\xi<T
$$

that is,

$$
\left(\left(K_{p, \alpha}-K_{q, \beta}\right)(1, \cdot) * g\right)(t)=0, \quad 0<t<T
$$

Since $g \not \equiv 0$, we apply the Titchmarsh convolution theorem (e.g., Titchmarsh [28]), so that there exists $t_{0}>0$ such that

$$
K_{p, \alpha}(1, t)=K_{q, \beta}(1, t), \quad 0<t<t_{0}
$$


Lemma 4 implies that $K_{p, \alpha}(1, t)$ and $K_{q, \beta}(1, t)$ are analytic in $t>0$, and so

$$
\sum_{n=1}^{\infty} \frac{1}{\lambda_{n} \rho_{n}}\left(1-E_{\alpha, 1}\left(-\lambda_{n} t^{\alpha}\right)\right)=\sum_{n=1}^{\infty} \frac{1}{\mu_{n} \sigma_{n}}\left(1-E_{\beta, 1}\left(-\mu_{n} t^{\beta}\right)\right), \quad t>0 .
$$

By the asymptotics of $E_{\alpha, 1}(-\eta)$ and $E_{\beta, 1}(-\eta)$ for large $\eta>0$ (e.g., Theorem 1.4 (pp.33-34) in [24]), we have

$$
E_{\alpha, 1}\left(-\lambda_{n} t^{\alpha}\right)=\frac{1}{\Gamma(1-\alpha)} \frac{1}{\lambda_{n} t^{\alpha}}+O\left(\frac{1}{t^{2 \alpha}}\right)
$$

and

$$
E_{\beta, 1}\left(-\mu_{n} t^{\beta}\right)=\frac{1}{\Gamma(1-\beta)} \frac{1}{\mu_{n} t^{\beta}}+O\left(\frac{1}{t^{2 \beta}}\right)
$$

for all large $t>0$. Hence

$$
\begin{aligned}
& \sum_{n=1}^{\infty} \frac{1}{\lambda_{n} \rho_{n}}-\frac{1}{\Gamma(1-\alpha)} \sum_{n=1}^{\infty} \frac{1}{\lambda_{n} \rho_{n}} \frac{1}{\lambda_{n} t^{\alpha}}+O\left(\frac{1}{t^{2 \alpha}}\right) \\
= & \sum_{n=1}^{\infty} \frac{1}{\mu_{n} \sigma_{n}}-\frac{1}{\Gamma(1-\beta)} \sum_{n=1}^{\infty} \frac{1}{\mu_{n} \sigma_{n}} \frac{1}{\mu_{n} t^{\beta}}+O\left(\frac{1}{t^{2 \beta}}\right)
\end{aligned}
$$

for large $t>0$. Letting $t \rightarrow \infty$, we obtain

$$
\sum_{n=1}^{\infty} \frac{1}{\lambda_{n} \rho_{n}}=\sum_{n=1}^{\infty} \frac{1}{\mu_{n} \sigma_{n}}
$$

Assume that $\alpha>\beta$. Then

$$
-\frac{1}{\Gamma(1-\alpha)} \sum_{n=1}^{\infty} \frac{1}{\lambda_{n} \rho_{n}} \frac{1}{\lambda_{n} t^{\alpha-\beta}}+O\left(\frac{1}{t^{2 \alpha-\beta}}\right)=-\frac{1}{\Gamma(1-\beta)} \sum_{n=1}^{\infty} \frac{1}{\mu_{n} \sigma_{n}} \frac{1}{\mu_{n}}+O\left(\frac{1}{t^{\beta}}\right)
$$

for large $t>0$. Letting $t \rightarrow \infty$, we obtain

$$
\frac{1}{\Gamma(1-\beta)} \sum_{n=1}^{\infty} \frac{1}{\mu_{n}^{2} \sigma_{n}}=0
$$

Since $\sigma_{n}=\left\|\psi_{n}\right\|^{2}>0$, this is impossible. Hence $\alpha \leq \beta$. By an entirely similar argument we see that $\alpha<\beta$ is impossible and so conclude that $\alpha=\beta$.

Now we move to complete the proof of the theorem. We see

$$
\sum_{n=1}^{\infty} \frac{1}{\lambda_{n} \rho_{n}} E_{\alpha, 1}\left(-\lambda_{n} t^{\alpha}\right)=\sum_{n=1}^{\infty} \frac{1}{\mu_{n} \sigma_{n}} E_{\alpha, 1}\left(-\mu_{n} t^{\alpha}\right), \quad t>0
$$

Now we can argue similarly to [3]. Using

$$
\left|\frac{1}{\lambda_{n} \rho_{n}} E_{\alpha, 1}\left(-\lambda_{n} t^{\alpha}\right)\right| \leq \frac{C}{\lambda_{n}}, \quad n \in \mathbb{N}, \quad t>0,
$$


we see that the series in $(3.1)$ are convergent uniformly in $[0, \infty)$. Therefore we can take the Laplace transforms termwise to have

$$
\sum_{n=1}^{\infty} \frac{1}{\lambda_{n} \rho_{n}} \int_{0}^{\infty} e^{-\zeta t} E_{\alpha, 1}\left(-\lambda_{n} t^{\alpha}\right) d t=\sum_{n=1}^{\infty} \frac{1}{\mu_{n} \sigma_{n}} \int_{0}^{\infty} e^{-\zeta t} E_{\alpha, 1}\left(-\mu_{n} t^{\alpha}\right) d t, \quad \zeta>0 .
$$

By formula (1.80) (p.21) in [24], we obtain

$$
\sum_{n=1}^{\infty} \frac{1}{\lambda_{n} \rho_{n}} \frac{\zeta^{\alpha-1}}{\zeta^{\alpha}+\lambda_{n}}=\sum_{n=1}^{\infty} \frac{1}{\mu_{n} \sigma_{n}} \frac{\zeta^{\alpha-1}}{\zeta^{\alpha}+\mu_{n}}, \quad \zeta>0 .
$$

Dividing by $\zeta^{\alpha-1}$ and setting $\eta=\zeta^{\alpha}$, we have

$$
\sum_{n=1}^{\infty} \frac{1}{\lambda_{n} \rho_{n}} \frac{1}{\eta+\lambda_{n}}=\sum_{n=1}^{\infty} \frac{1}{\mu_{n} \sigma_{n}} \frac{1}{\eta+\mu_{n}}, \quad \eta>0
$$

Since $\lambda_{n} \sim n^{2}$ and $\mu_{n} \sim n^{2}$ for large $n \in \mathbb{N}$, we see that both sides of (3.2) are convergent uniformly in any compact set in $\mathbb{C} \backslash\left(\left\{-\lambda_{n}\right\}_{n \in \mathbb{N}} \cup\left\{-\mu_{n}\right\}_{n \in \mathbb{N}}\right)$ and are analytic in $\mathbb{C} \backslash\left(\left\{-\lambda_{n}\right\}_{n \in \mathbb{N}} \cup\left\{-\mu_{n}\right\}_{n \in \mathbb{N}}\right)$.

Assume that $\lambda_{m} \notin\left\{\mu_{n}\right\}_{n \in \mathbb{N}}$ for $m \in \mathbb{N}$. Then we can choose a small circle $C_{m}$ centered at $-\lambda_{m}$ and $\left\{-\mu_{n}\right\}_{n \in \mathbb{N}}$ is not included in the disk centered at $-\lambda_{n}$ bounded by $C_{m}$. Integrating on $C_{m}$ and applying the Cauchy theorem, we have

$$
\frac{2 \pi \sqrt{-1}}{\lambda_{m} \rho_{m}}=0
$$

which is impossible. Hence $\lambda_{m} \in\left\{\mu_{n}\right\}_{n \in \mathbb{N}}$ for each $m$. Similarly $\mu_{m} \in\left\{\lambda_{n}\right\}_{n \in \mathbb{N}}$ for each $m \in \mathbb{N}$. Therefore

$$
\lambda_{n}=\mu_{n}, \quad n \in \mathbb{N} .
$$

By (3.2), we have

$$
\sum_{n=1}^{\infty}\left(\frac{1}{\lambda_{n} \rho_{n}}-\frac{1}{\lambda_{n} \sigma_{n}}\right) \frac{1}{\eta+\lambda_{n}}=0, \quad \eta \in \mathbb{C} \backslash\left\{-\lambda_{n}\right\}_{n \in \mathbb{N}}
$$

Again integrating on $C_{m}$, we obtain

$$
\frac{2 \pi \sqrt{-1}}{\lambda_{n}}\left(\frac{1}{\rho_{n}}-\frac{1}{\sigma_{n}}\right)=0
$$

that is,

$$
\rho_{n}=\sigma_{n}, \quad n \in \mathbb{N} .
$$


Now, using (3.3) and (3.4), we apply the Gel'fand-Levitan theory (e.g., Gel'fand and Levitan [4]), and we can obtain $p(x)=q(x)$ for $0<x<1$. The application is similar to [3], [22], [27], and so we omit the details. Thus the proof of Theorem 2 is complete.

\section{Applichtion of the Representation Formula}

The representation formula Proposition 3 is useful for qualitative analyses of fractional equations. Here we explain one application.

We let $0<\alpha<1$ and we fix $p \in C[0,1], \leq 0$ on $[0,1]$. Let

$$
\left\{\begin{array}{l}
\partial_{t}^{\alpha} u(x, t)=\partial_{x}^{2} u(x, t)+p(x) u(x, t), \quad 0<x<1,0<t<T \\
u(0, t)=\partial_{x} u(0, t)=0, \quad 0<t<T \\
u \in H_{\alpha}\left(0, T ; L^{2}(0,1)\right) .
\end{array}\right.
$$

Then we are interested in the question: can we conclude $u(x, t)=0$ for $0<x<1$ and $0<t<T ?$

This is a kind of unique continuation property under the assumption $u \in H_{\alpha}\left(0, T ; L^{2}(0,1)\right)$ which can be interpreted as that an initial value of $u$ is zero. This kind of unique continuation was proved by Cheng, Lin and Nakamura [2] for $\alpha=\frac{1}{2}$, Lin and Nakamura [17] for $\alpha \in(0,1)$ and Lin and Nakamura [18] for $\alpha \in(0,1) \cup(1,2)$ for general time-fractional partial differential equations. Their proofs are based on the techniques of pseudo-differential operators.

For $\alpha=1$, we can prove the unique continuation without any information of initial conditions, and the corresponding unique continuation is proved for a one-dimensional timefractional equation by $\mathrm{Li}$ and Yamamoto [15]. More precisely, if $u$ is in a suitable class and satisfies

$$
\left\{\begin{array}{l}
\partial_{t}^{\alpha} u(x, t)=\partial_{x}^{2} u(x, t), \quad 0<x<1,0<t<T, \\
u(0, t)=\partial_{x} u(0, t)=0, \quad 0<t<T
\end{array}\right.
$$

then $u(x, t)=0$ for $0<x<1$ and $0<t<T$.

However such unique continuation not requiring any initial conditions, is not known for general case in multidimensions.

In this section, for the one-dimensional case (4.1), we provide a simpler proof than [2, 17, 18, which relies on the representation formula Proposition 3.

\section{Proposition 5.}


Let $u \in H_{\alpha}\left(0, T ; L^{2}(0,1)\right) \cap L^{2}\left(0, T ; H^{2}(0,1)\right)$ satisfy (4.1) and $\partial_{x} u(1, \cdot) \in H_{\alpha}(0, T)$. Then $u(x, t)=0,0<x<1,0<t<T$.

By the definition of $H_{\alpha}(0, T)$ given in Section 1 , if $0<\alpha<\frac{1}{2}$, then $H_{\alpha}(0, T)=H^{\alpha}(0, T)$ and in (4.1) the condition $u \in H_{\alpha}\left(0, T ; L^{2}(0,1)\right)$ does not require anything for the behavior of the solution $u$ near $t=0$. In other words, we need not pose any conditions at $t=0$ to $u$.

It seems that we can remove a condition $\partial_{x} u(1, \cdot) \in H_{\alpha}(0, T)$, but we here omit the details.

\section{Proof.}

We set $g:=\partial_{x} u(1, \cdot) \in H_{\alpha}(0, T)$. Then $u$ satisfies $(1.4)$ and $u(0, t)=0,0<t<T$. Lemma 4 (ii) implies

$$
\left(K_{p, \alpha}(0, \cdot) * g\right)(t)=0, \quad 0<t<T \text {. }
$$

By the Titchmarsh theorem on the convolution (e.g., [28]), there exist $t_{1}, t_{2} \geq 0$ such that

$$
\left\{\begin{array}{l}
t_{1}+t_{2}=T \\
K_{p, \alpha}(0, t)=0, \quad 0 \leq t \leq t_{1}, \\
g(t)=0, \quad 0 \leq t \leq t_{2} .
\end{array}\right.
$$

Assuming that $t_{1}>0$, we will derive a contradiction, which proves $t_{1}=0$, that is, $g=0$ in $(0, T)$. The argument is similar to the proof of Theorem 2.

The analyticity of $K_{p, \alpha}(0, t)$ in $t>0$ yields $K_{p, \alpha}(0, t)=0$ for all $t>0$.

Since $\lim _{t \rightarrow \infty} E_{\alpha, 1}\left(-\lambda_{n} t^{\alpha}\right)=0$ (e.g., Theorem 1.6 (p.35) in [24]), we have

$$
\lim _{t \rightarrow \infty} K_{p, \alpha}(0, t)=\sum_{n=1}^{\infty} \frac{\varphi_{n}(0)}{\lambda_{n} \rho_{n}}=0 .
$$

Hence

$$
\sum_{n=1}^{\infty} \frac{\varphi_{n}(0)}{\lambda_{n} \rho_{n}} E_{\alpha, 1}\left(-\lambda_{n} t^{\alpha}\right)=0, \quad t>0 .
$$

This series is convergent in $L^{\infty}(0, \infty)$ and so we can take the Laplace transform term by term. In view of formula (1.80) (p.21) in [24], we obtain

$$
\sum_{n=1}^{\infty} \frac{\varphi_{n}(0)}{\lambda_{n} \rho_{n}} \frac{z^{\alpha-1}}{z^{\alpha}+\lambda_{n}}=0, \quad \operatorname{Re} z>0 .
$$


Then, dividing by $z^{\alpha-1}$ and setting $\eta=z^{\alpha}$, we have

$$
\sum_{n=1}^{\infty} \frac{\varphi_{n}(0)}{\lambda_{n} \rho_{n}} \frac{1}{\eta+\lambda_{n}}=0, \quad \operatorname{Re} \eta>0 .
$$

Similarly to (2.10), we can verify

$$
\sum_{n=1}^{\infty} \frac{\varphi_{n}(0)}{\lambda_{n} \rho_{n}}<\infty
$$

and so we can continue analytically in $\eta$ as much as possible to obtain

$$
\sum_{n=1}^{\infty} \frac{\varphi_{n}(0)}{\lambda_{n} \rho_{n}} \frac{1}{\eta+\lambda_{n}}=0, \quad \eta \in \mathbb{C} \backslash\left\{-\lambda_{n}\right\}_{n \in \mathbb{N}} .
$$

Choosing a small circle $\Gamma_{1}$ centered at $-\lambda_{1}$ such that the interior of the disk bounded by $\Gamma_{1}$ does not contain $-\lambda_{n}$ with $n \geq 2$ and integrating on $\Gamma_{1}$, in terms of the Cauchy theorem, we see

$$
\frac{\varphi_{1}(0)}{\lambda_{1} \rho_{1}} 2 \pi \sqrt{-1}=0
$$

that is, $\varphi_{1}(0)=0$. Since $\frac{d^{2} \varphi_{1}}{d x^{2}}(x)+\left(p(x)+\lambda_{1}\right) \varphi_{1}(x)=0,0<x<1$ and $\frac{d \varphi_{1}}{d x}(0)=0$, we have $\varphi_{1}(x)=0$ for all $0<x<1$, which is impossible.

Then we can conclude that $t_{1}=0$. By (4.2), we reach $g(t)=0$ for $0<t<T$. Thus the proof of Proposition 5 is complete.

\section{ACKNOWLEDGMENTS}

The first author was supported in part by the National Science Foundation through award DMS-1620138.

The second author was supported by Grant-in-Aid for Scientific Research (S) $15 \mathrm{H} 05740$ of Japan Society for the Promotion of Science and by The National Natural Science Foundation of China (no. 11771270, 91730303). This work was prepared with the support of the "RUDN University Program 5-100".

\section{REFERENCES}

[1] R.A. Adams, Sobolev Spaces, Academic Press, New York, 1975.

[2] J. Cheng, C. Lin, and G. Nakamura, Unique continuation property for the anomalous diffusion and its application, Journal of Differential Equations 254 (2013) 3715-3728.

[3] J. Cheng, J. Nakagawa, M. Yamamoto, and T. Yamazaki, Uniqueness in an inverse problem for a one-dimensional fractional diffusion equation, Inverse Problems 25 (2009), 115002 
[4] I.M. Gel'fand and B.M. Levitan, On the determination of a differential equation from its spectral function, Amer. Math. Soc. Tanslation 1 (1955) 253-304.

[5] R. Gorenflo, A.A. Kilbas, F. Mainardi and S. V. Rogosin, Mittag-Leffler Functions, Related Topics and Applications, Springer-Verlag, Berlin, 2014.

[6] R. Gorenflo, Y. Luchko and M. Yamamoto, Time-fractional diffusion equation in the fractional Sobolev spaces, Fractional Calculus and Applied Analysis 18 (2015) 799-820.

[7] B. Jin and W. Rundell, An inverse problem for a one-dimensional time-fractional diffusion problem, Inverse Problems 28 (2012) 75010-75028(19pp).

[8] X. Jing and M. Yamamoto, Uniqueness of the coefficient for a time-fractional diffusion equation, preprint.

[9] Y. Kian and M. Yamamoto, On existence and uniqueness of solutions for semilinear fractional wave equations, Fract. Calc. Appl. Anal. 20 (2017) 117-138.

[10] A. Kubica, K. Ryszewska, and M. Yamamoto, Introduction to a Theory of Time-fractional Partial Differential Equations, Springer Japan, Tokyo, 2020.

[11] A. Kubica and M. Yamamoto, Initial-boundary value problems for fractional diffusion equations with time-dependent coefficients, Fract. Calc. Appl. Anal. 21 (2018) 276-311.

[12] B.M.Levitan and I.S.Sargsjan, Introduction to Spectral Theory, American Mathematical Society, Providence, Rhode Island 1975.

[13] G. Li, D. Zhang, X. Jia and M. Yamamoto, Simultaneous inversion for the space-dependent diffusion coefficient and the fractional order in the time-fractional diffusion equation, Inverse Problems 29 (2013) 065014, $36 \mathrm{pp}$.

[14] Z. Li, Y. Liu and M. Yamamoto, Inverse problems of determining parameters of the fractional partial differential equations, Handbook of Fractional Calculus with Applications (ed: J.A. Tenreiro Machado, A. N. Kochubei and Y. Luchko) Vol. 2, pp. 431-442, De Gruyter, Berlin, 2019.

[15] Z. Li and M. Yamamoto, Unique continuation principle for the one-dimensional time-fractional diffusion equation, Fract. Calc. Appl. Anal. 22 (2019) 644-657.

[16] Z. Li and M. Yamamoto, Inverse problems of determining coefficients of the fractional partial differential equations. Handbook of fractional calculus with applications. Vol. 2, pp. 443-464, De Gruyter, Berlin, 2019.

[17] C.-L. Lin and G. Nakamura, Unique continuation property for anomalous slow diffusion equation, Communications in Partial Differential Equations 41 (2016) 749-758.

[18] C.-L. Lin and G. Nakamura, Unique continuation property for multi-terms time fractional diffusion equations, Math. Ann. 373 (2019) 929-952.

[19] Y. Liu, Z. Li, and M. Yamamoto, Inverse problems of determining sources of the fractional partial differential equations. Handbook of fractional calculus with applications. Vol. 2, pp. 411-429, De Gruyter, Berlin, 2019. 
[20] Y. Luchko, Initial-boundary-value problems for the generalized multi-term time-fractional diffusion equation, J. Math. Anal. Appl. 374 (2011) 538-548.

[21] R. Metzler and J. Klafter, The random walkfs guide to anomalous diffusion: a fractional dynamics approach, Phyics Reports 339 (2000) 1-77.

[22] R. Murayama, The Gel'fand-Levitan theory and certain inverse problems for the parabolic equation, J. Fac. Sci. The Univ. Tokyo Section IA, Math. 28 (1981) 317-330.

[23] A. Pierce, Unique identification of eigenvalues and coefficients in a parabolic problem, SIAM J. Control and Optim. 17 (1979) 494-499.

[24] I. Podlubny, Fractional Differential Equations, Academic Press, San Diego, 1999.

[25] W. Rundell and M. Yamamoto, Recovery of a potential in a fractional diffusion equation, 2018, arXiv:1811.05971v1

[26] K. Sakamoto and M. Yamamoto, Initial value/boundary value problems for fractional diffusion-wave equations and applications to some inverse problems, J. Math. Anal. Appl. 382 (2011) 426-447.

[27] T. Suzuki and R. Murayama, A uniqueness theorem in an identification problem for coefficients of parabolic equations, Proc. Japan Acad. Ser. A 56 (1980) 259-263.

[28] E.C. Titchmarsh, The zeros of certain integral functions, Proc. London. Math. Soc. 25 (1926) 283-302.

[29] T. Wei and X.B. Yan, Uniqueness for identifying a space-dependent zeroth-order coefficient in a timefractional diffusion-wave equation from a single boundary point measurement, preprint.

[30] M. Yamamoto, Weak solutions to non-homogeneous boundary value problems for time-fractional diffusion equations, J. Math. Anal. Appl. 460 (2018) 365-381. 